\title{
Soft chemical routes to electrochemically active iron phosphates
}

Prashanth Sandineni ${ }^{\mathrm{a}}$, Hooman Yaghoobnejad Asla ${ }^{\mathrm{a}}$, Nikolay Gerasimchuk ${ }^{\mathrm{b}}$, Kartik Ghosh ${ }^{\mathrm{c}}$, and Amitava Choudhury ${ }^{\mathrm{a}, *}$

a Department of Chemistry, Missouri University of Science and Technology, Rolla, MO 65409, USA.

bepartment of Chemistry, Missouri State University, Springfield, MO 65897, USA.

${ }^{c}$ Department of Physics, Astronomy and Materials Science and Center for Applied Science and Engineering, Missouri State University, Springfield, Missouri 65897, USA. 


\section{Coin Cell fabrication and electrochemical test:}

To the finely ground active material Poly-vinylidene fluoride (PVDF) was added as the binder and an optimum amount of N-methyl-2-pyrrolidone (NMP) was added to dissolve the PVDF. The resulting slurry was further ball milled for another 20 minutes to obtain a homogeneous viscous mixture. The ratio of the active material, conducting carbon, and the binder in the cathode mix was 75: 15: 10. The cathode mix was then spread as a film of uniform thickness with the help of a glass rod onto a flat sheet of carbon-coated aluminum current collector and transferred into a vacuum oven and dried overnight at $85^{\circ} \mathrm{C}$.

For electrochemical tests, CR2032 type coin cells were fabricated. The composite cathode film was cut into circular disks (3/8 inch diameter) with 4.0-5.0 mg of active material loading and transferred into an argon filled glove box with an oxygen concentration below $2.0 \mathrm{ppm}$. The cathode disk and $\mathrm{Li}$ anode $(0.75 \mathrm{~mm}$ thickness $\mathrm{Li}$ ribbon cut into circular disk) were assembled in the coin cell casing with a Celgard $® 2325$ circular sheet placed between the two electrodes as the separator. The electrolyte, $1 \mathrm{M}$ solution of $\mathrm{LiPF}_{6}$ in DMC-EC (1:1) was then added and the cell was sealed with a coin cell crimper. The prepared cells were aged for equilibration for about 12 hours before electrochemical testing. Similarly $\mathrm{Na}$ ion batteries were also made using $\mathrm{Na}$ anode and $1 \mathrm{M}$ solution of $\mathrm{NaClO}_{4}$ in $\mathrm{DMC}-\mathrm{EC}(1: 1)$ was used as the electrolyte.

Cyclic voltammograms were obtained using a PAR EG\&G potentiostat/galvanostat model 273 in the potential range of $1.5-4.0 \mathrm{~V}\left(\mathrm{vs} . \mathrm{Li} / \mathrm{Li}^{+}\right)$and $\left(\mathrm{vs} . \mathrm{Na} / \mathrm{Na}^{+}\right)$with a scan rate of $0.05 \mathrm{mV} \mathrm{s}$. Voltage composition profiles were obtained using galvanostatic charge/discharge experiments on an Arbin Instruments battery tester, model BT2043, on the same potential limits as CV at various C-rates. 


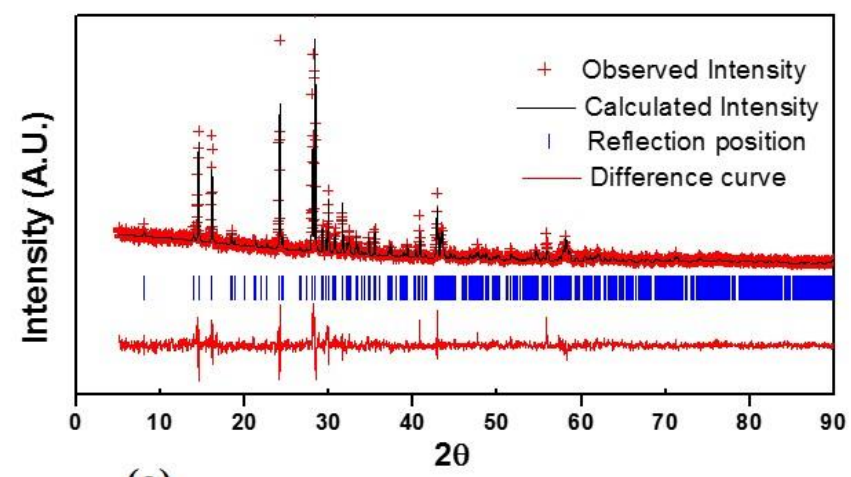

(a)

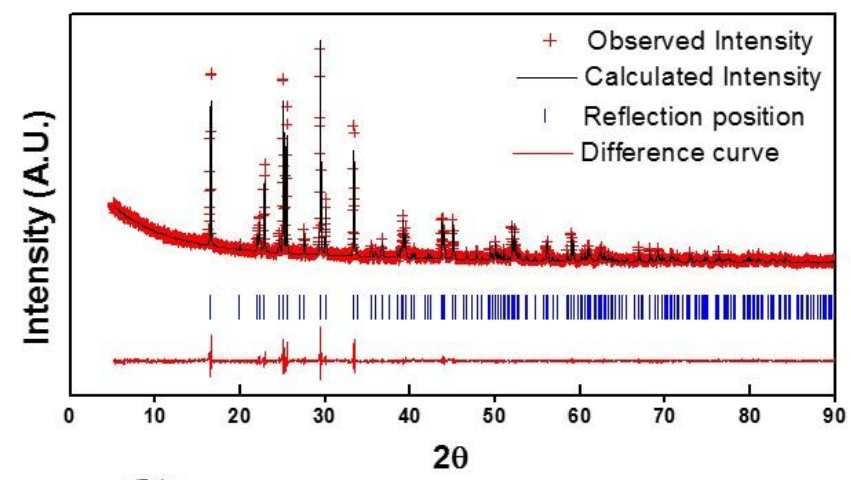

(b)

Figure S1. Rietveld refinement of low-resolution powder X-ray diffraction patterns of (a) $\mathrm{NaFe}\left(\mathrm{HPO}_{4}\right)_{2}(\lambda=1.54059 \AA, \mathrm{Rw}=12.858 \%)$ and (b) $\mathrm{Li}_{2} \mathrm{Fe}\left(\mathrm{H}_{0.5} \mathrm{PO}_{4}\right)_{2}-\mathrm{HT} \quad(\lambda=$ $1.54059 \AA$, $\mathrm{Rw}=12.246 \%$ ) 

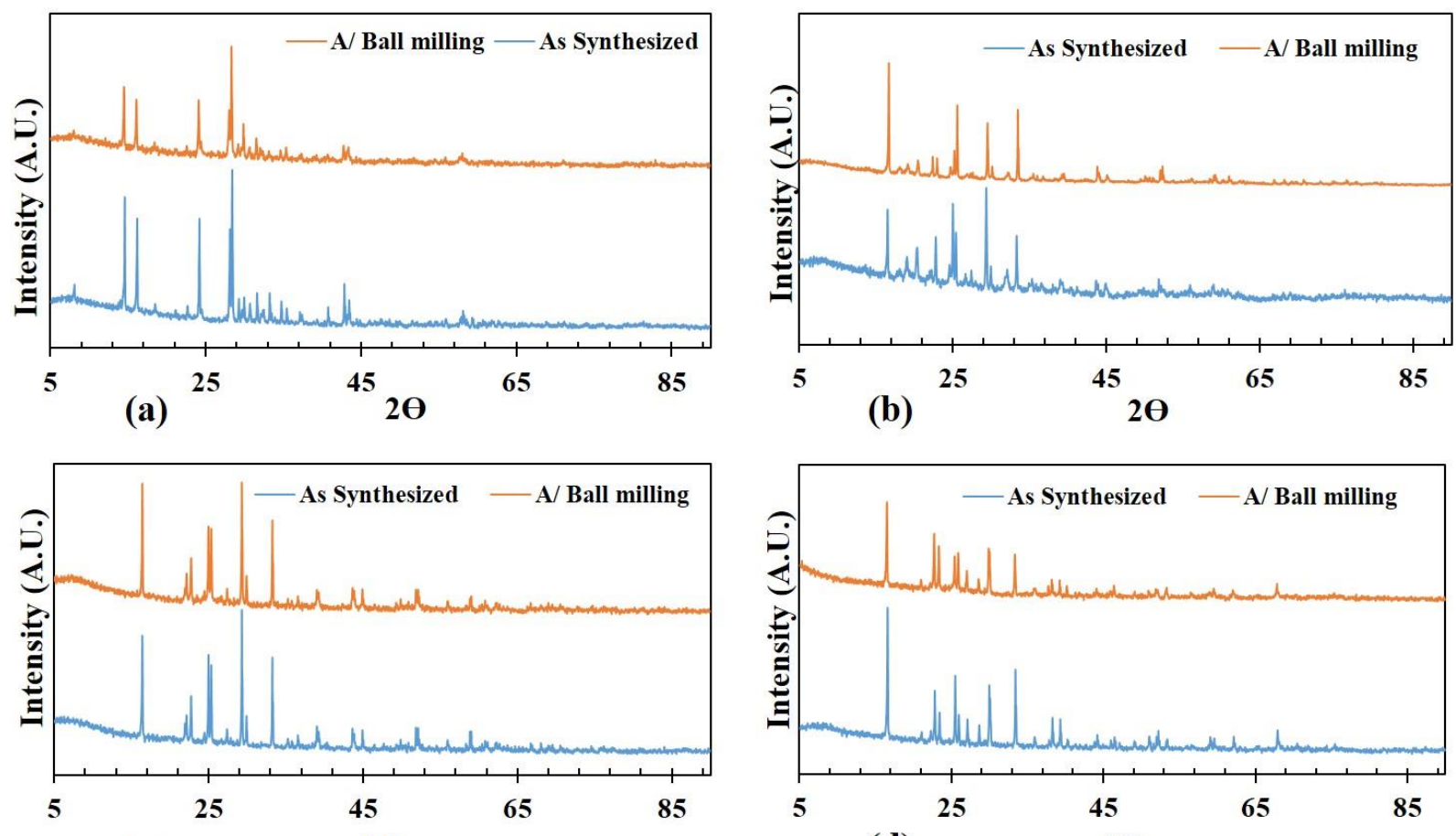

(c)

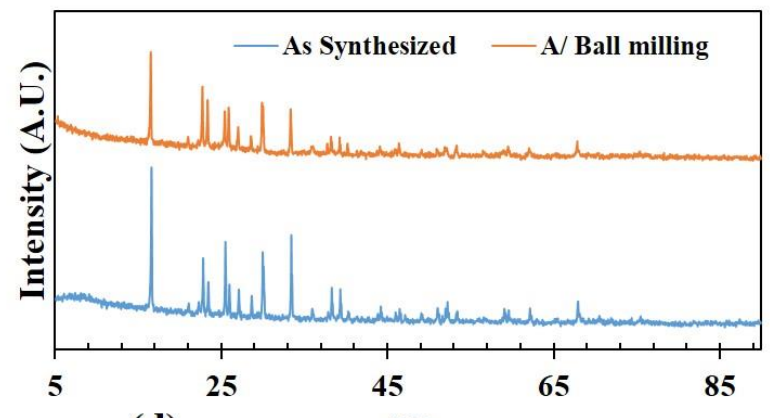

(d)

$2 \theta$

Figure S2. Powder XRDs of As Synthesized and After Ball milling samples for (a) $\mathrm{NaFe}\left(\mathrm{HPO}_{4}\right)_{2}$; (b) $\mathrm{Li}_{2} \mathrm{Fe}\left(\mathrm{H}_{0.5} \mathrm{PO}_{4}\right)_{2}-\mathrm{IEX}$, (c) $\mathrm{Li}_{2} \mathrm{Fe}\left(\mathrm{H}_{0.5} \mathrm{PO}_{4}\right)_{2}-\mathrm{HT}$; (d) $\mathrm{Li}_{3} \mathrm{Fe}\left(\mathrm{PO}_{4}\right)_{2}$. 


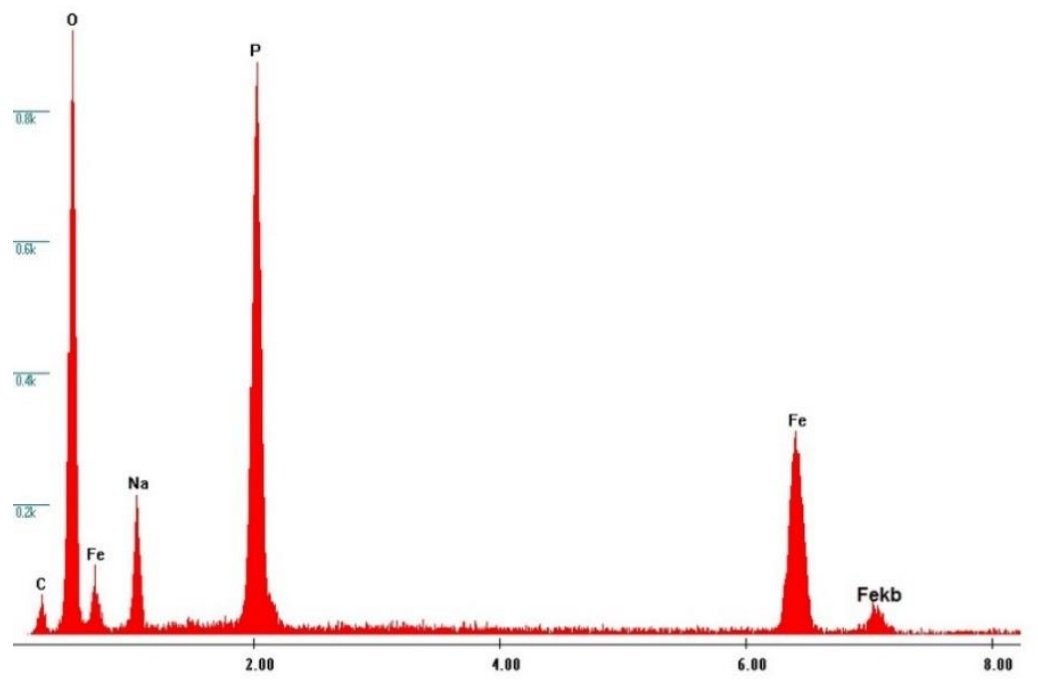

(a)

\begin{tabular}{crr} 
Element & Wt $\%$ & \multicolumn{1}{c}{ At \% } \\
\hline B K & 0.00 & 0.00 \\
C K & 8.36 & 14.93 \\
O K & 39.88 & 53.50 \\
F K & 0.00 & 0.00 \\
NaK & 9.50 & 8.87 \\
P K & 20.94 & 14.51 \\
FeK & 21.32 & 8.19 \\
Total & 100.00 & 100.00
\end{tabular}

(b)

Figure S3. EDS spectra of $\mathrm{NaFe}\left(\mathrm{HPO}_{4}\right)_{2}$. 

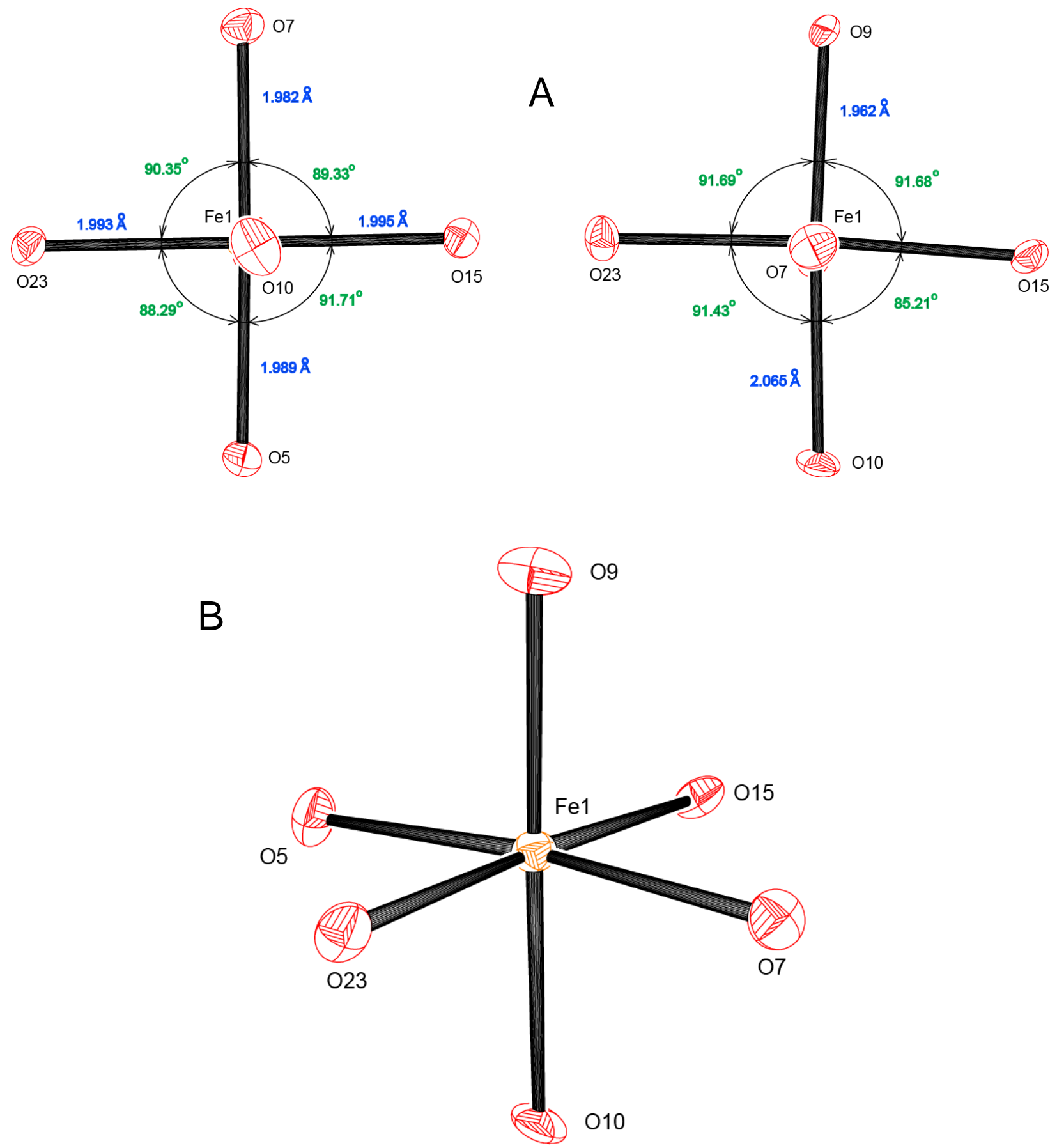

Figure S4. Environment of Fe1 center in the structure: $\mathbf{A}$ - two orthogonal projections, $\mathbf{B}-$ prospective view showing small distortions of octahedral polyhedron of the metal center. 

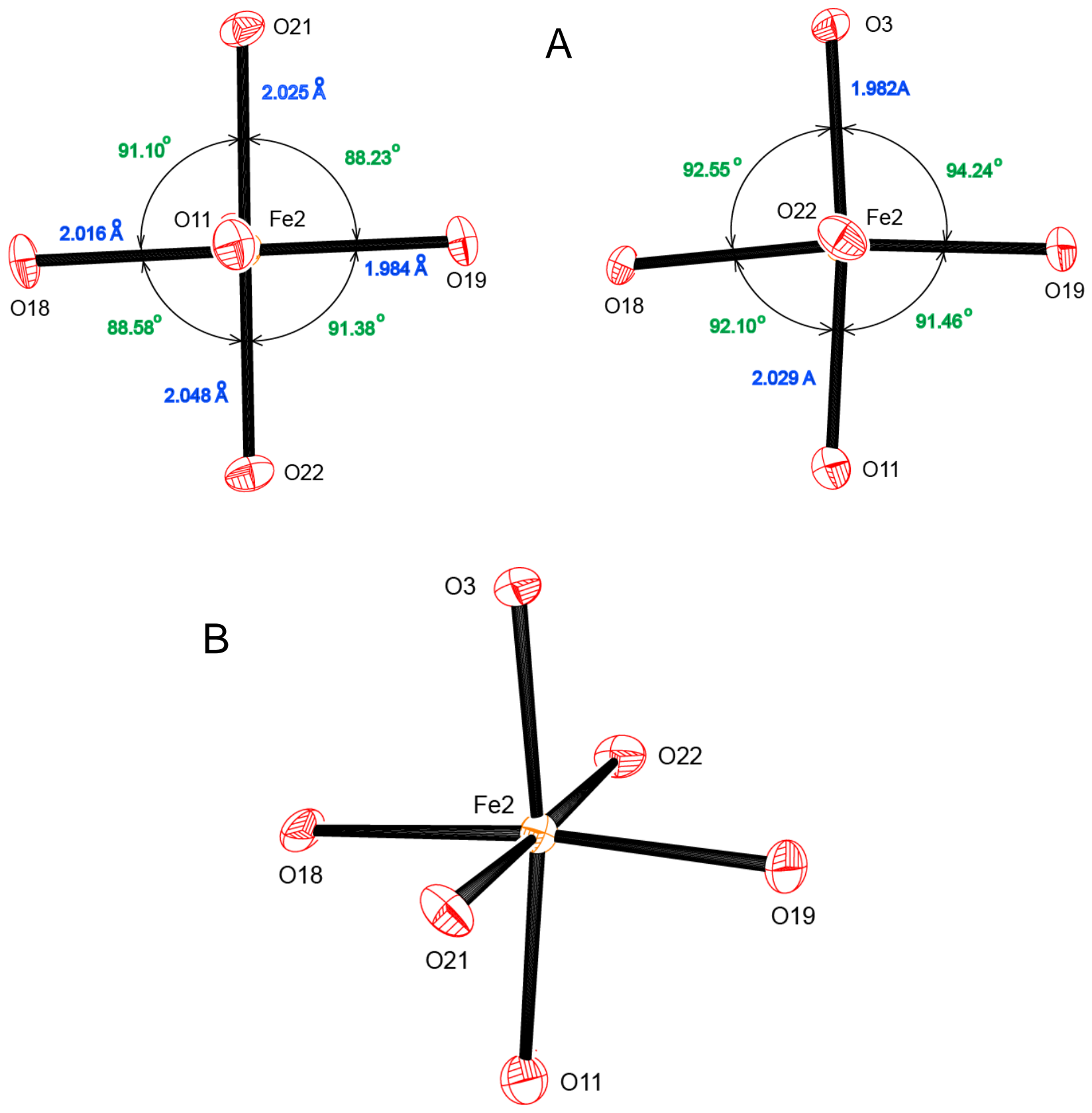

Figure S5. Environment of Fe2 center in the structure: $\mathbf{A}$ - two orthogonal projections, $\mathbf{B}$ prospective view showing small distortions of octahedral polyhedron of the metal center. 

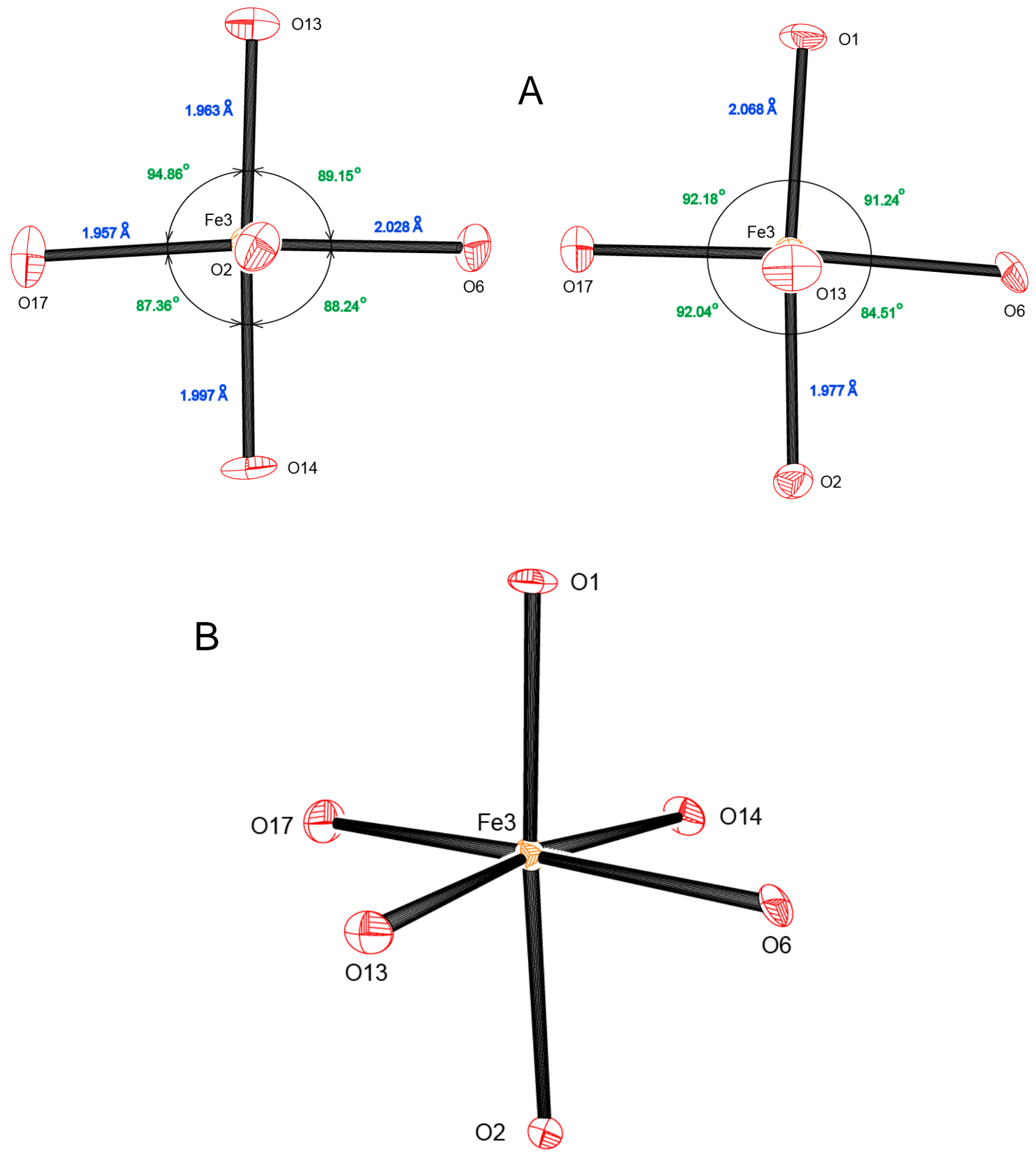

Figure S6. Environment of Fe3 center in the structure: A - two orthogonal projections, B prospective view showing small distortions of octahedral polyhedron of the metal center. 


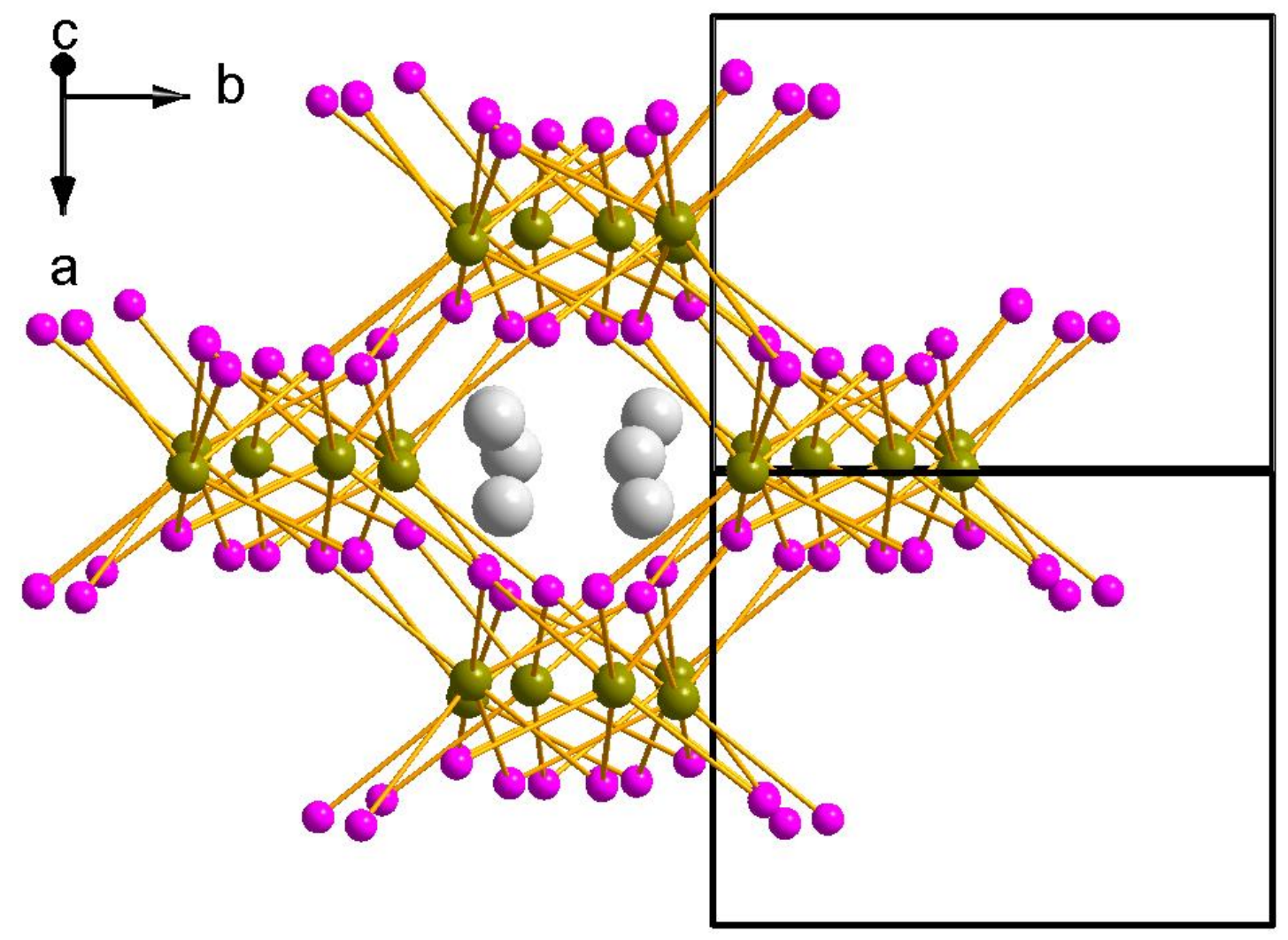

Figure S7. View of the structure of $\mathrm{NaFe}\left(\mathrm{HPO}_{4}\right)_{2}$ along [ $\left[\begin{array}{lll}1 & 0 & 7\end{array}\right]$ direction showing the channel filled with sodium ions. Note that oxygen atoms have been removed from the structure for clarity in visualization. 


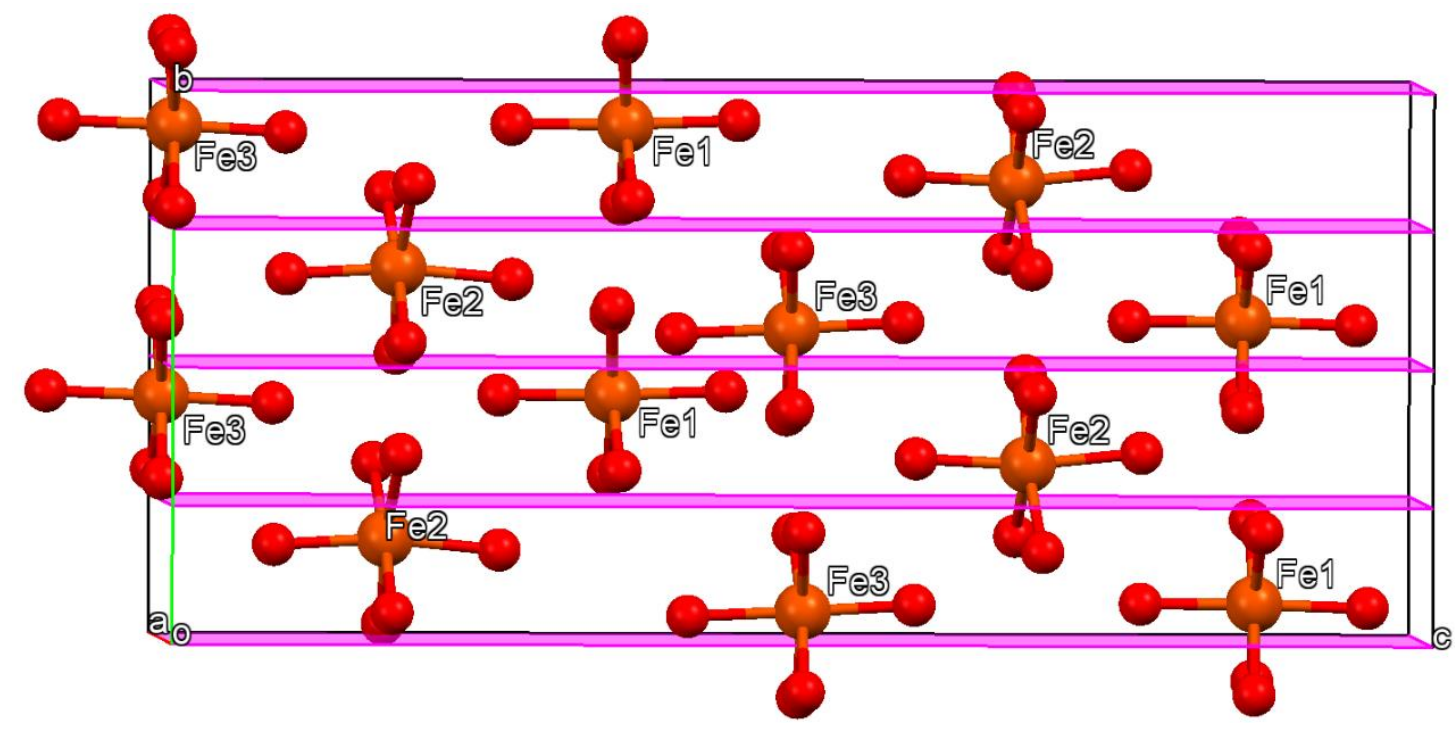

Figure S8. Structure of $\mathrm{NaFe}\left(\mathrm{HPO}_{4}\right)_{2}$ viewed along the a-axis showing only the Fe-centered octahedra. Purple planes indicate glide planes. Note the orientation of the crystallographically distinct Fe-octahedra display lack of center of symmetry in the structure. 


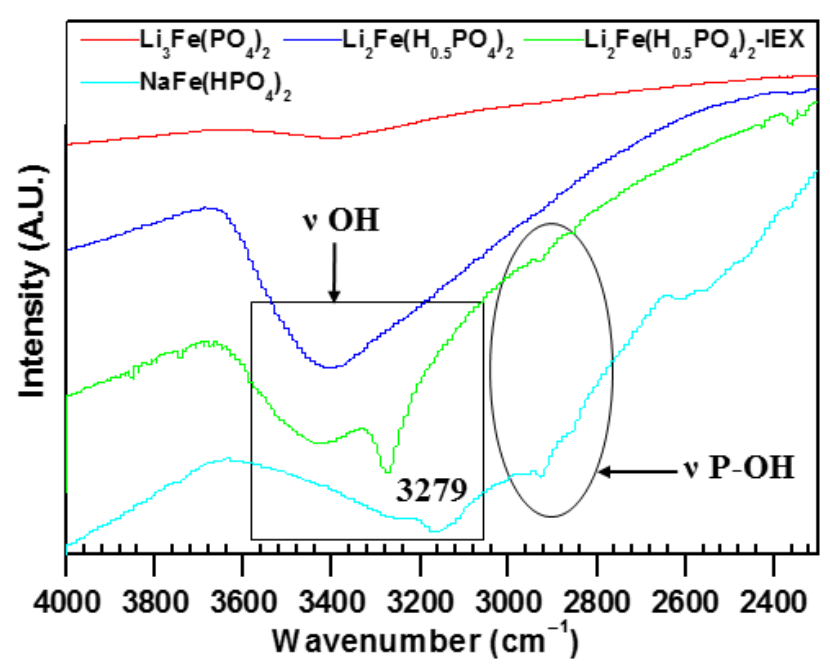

(a)

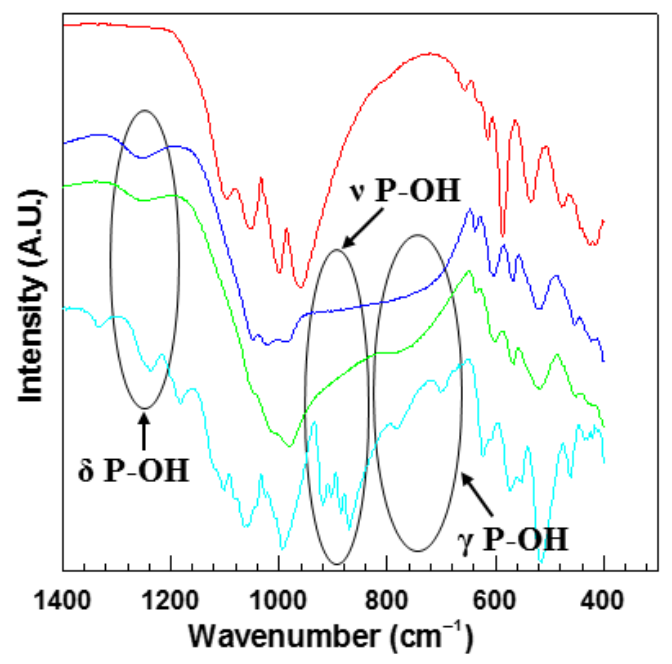

(b)

Figure S9. FT-IR spectra for the four compounds. 


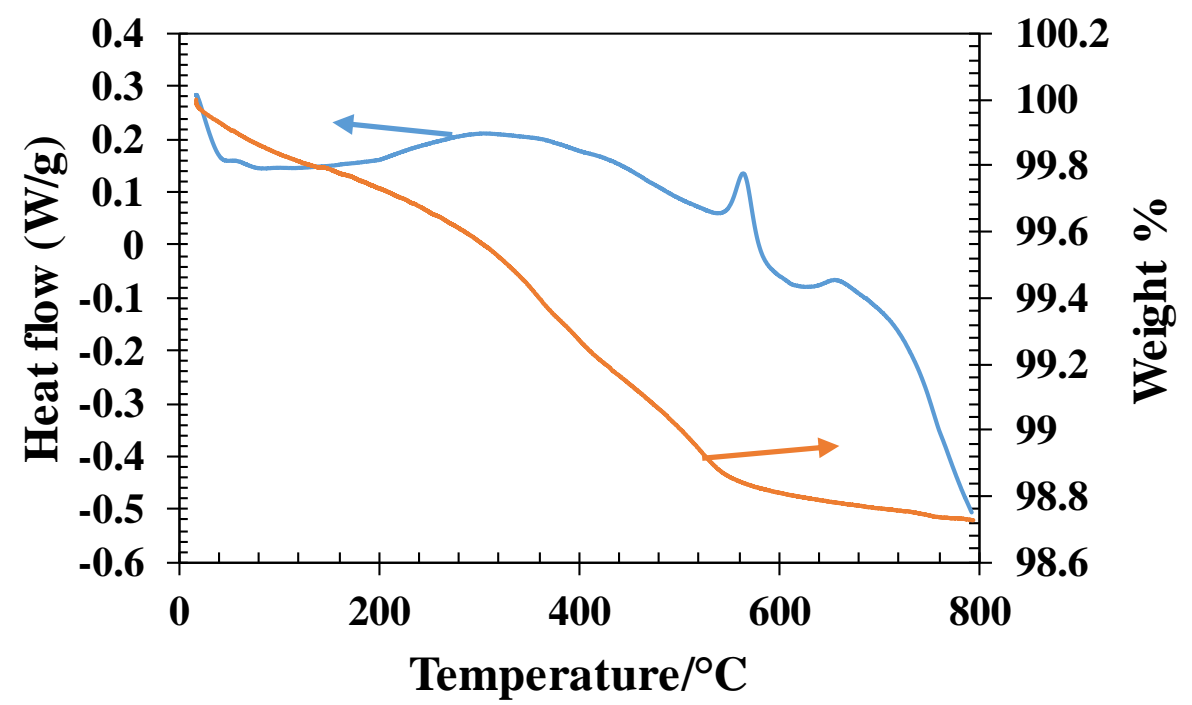

Figure S10. Simultaneous TGA/DSC curves for $\mathrm{Li}_{3} \mathrm{Fe}\left(\mathrm{PO}_{4}\right)_{2}$. 


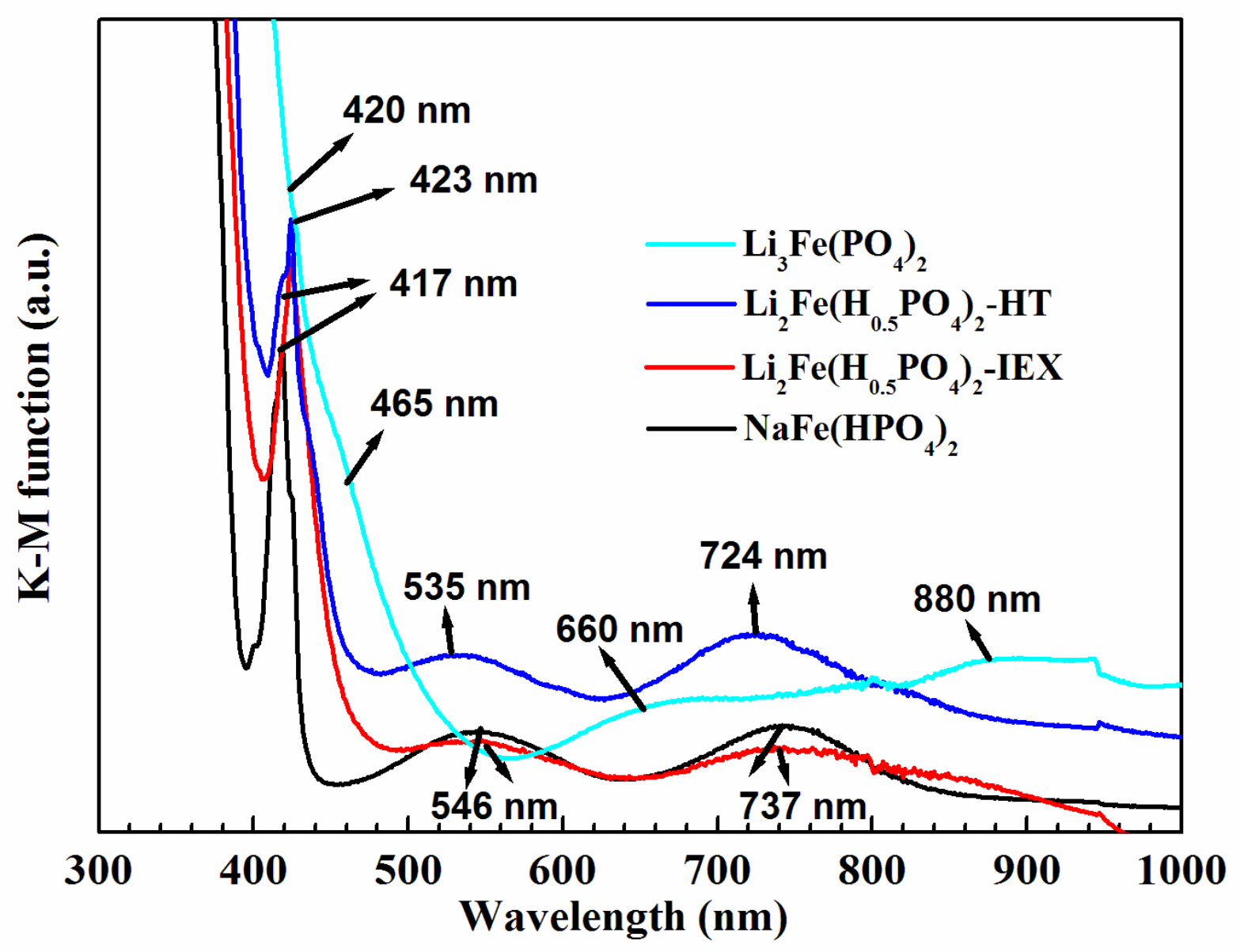

Figure S11: DRS spectra for the compounds. 


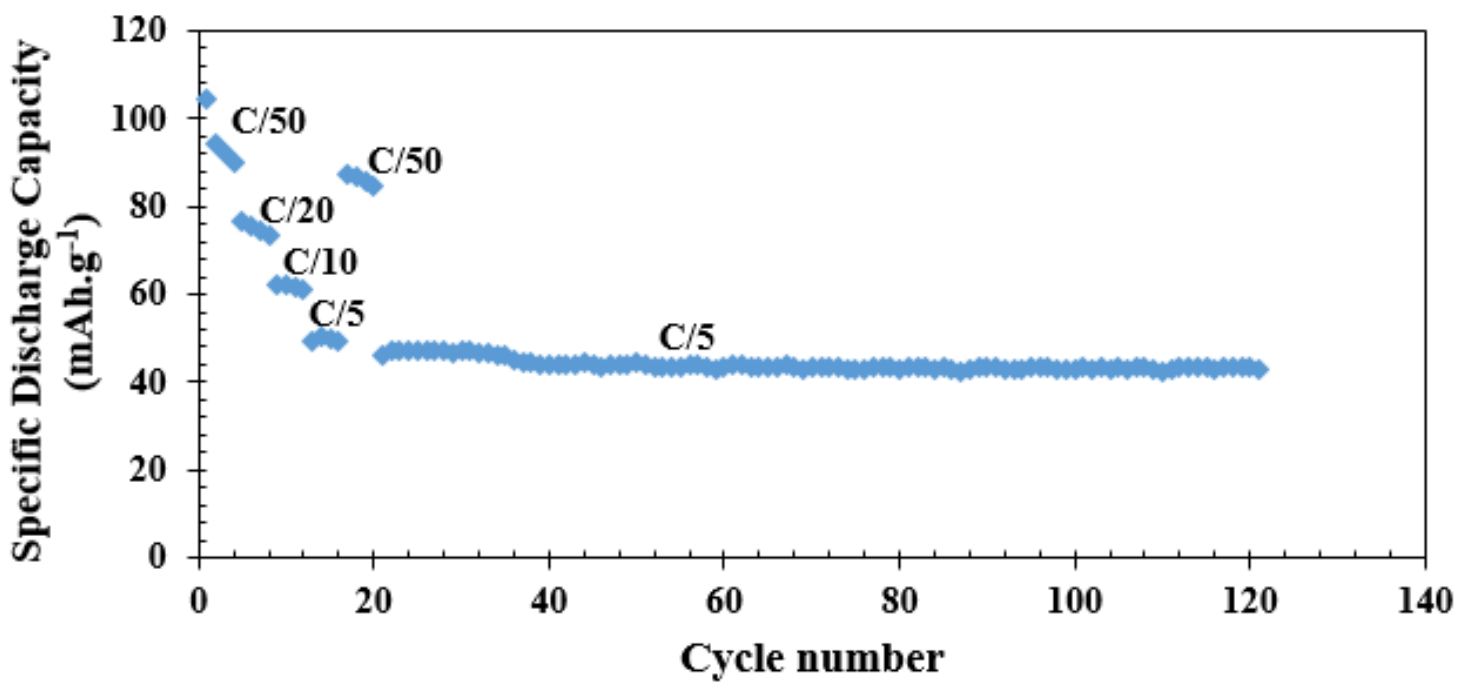

Figure S12. Discharge profile showing 100 cycles at C/5 rate after 20 cycles (four each of $\mathrm{C} / 50, \mathrm{C} / 20, \mathrm{C} / 10, \mathrm{C} / 5)$ for $\mathrm{Li}_{2} \mathrm{Fe}\left(\mathrm{H}_{0.5} \mathrm{PO}_{4}\right)_{2}$-IEX. 


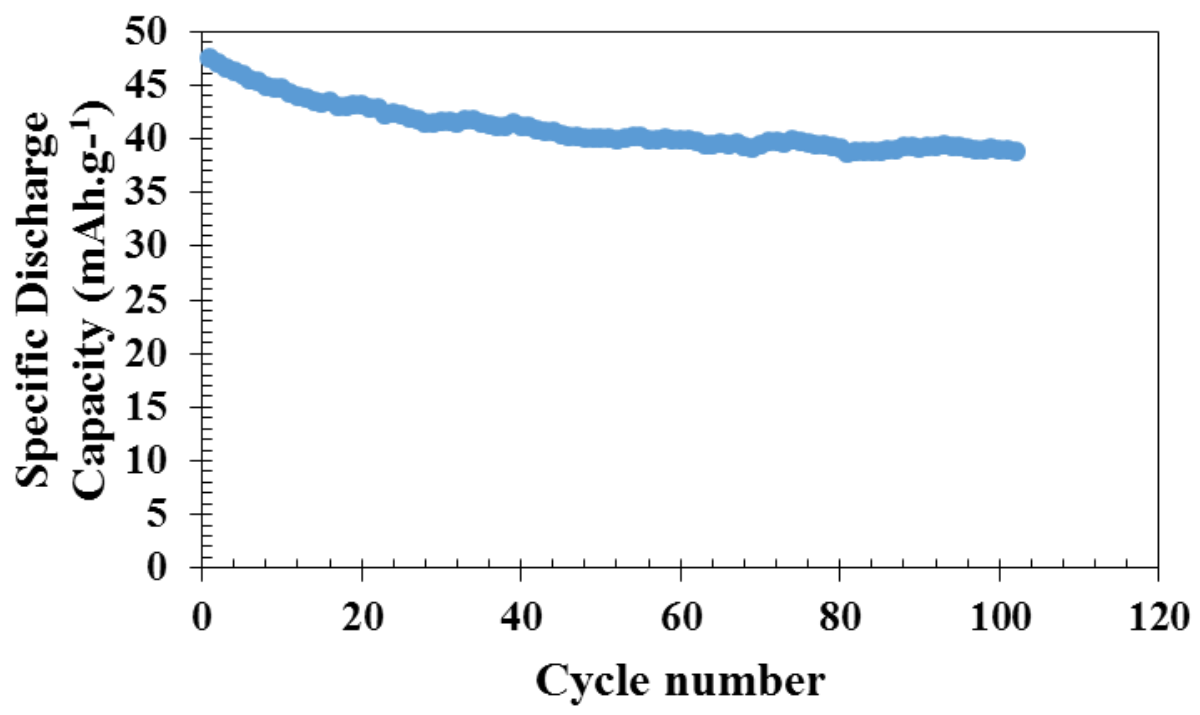

Figure S13. Discharge profile showing 102 cycles at $\mathrm{C} / 5$ rate for $\mathrm{Li}_{3} \mathrm{Fe}\left(\mathrm{PO}_{4}\right)_{2}$. 


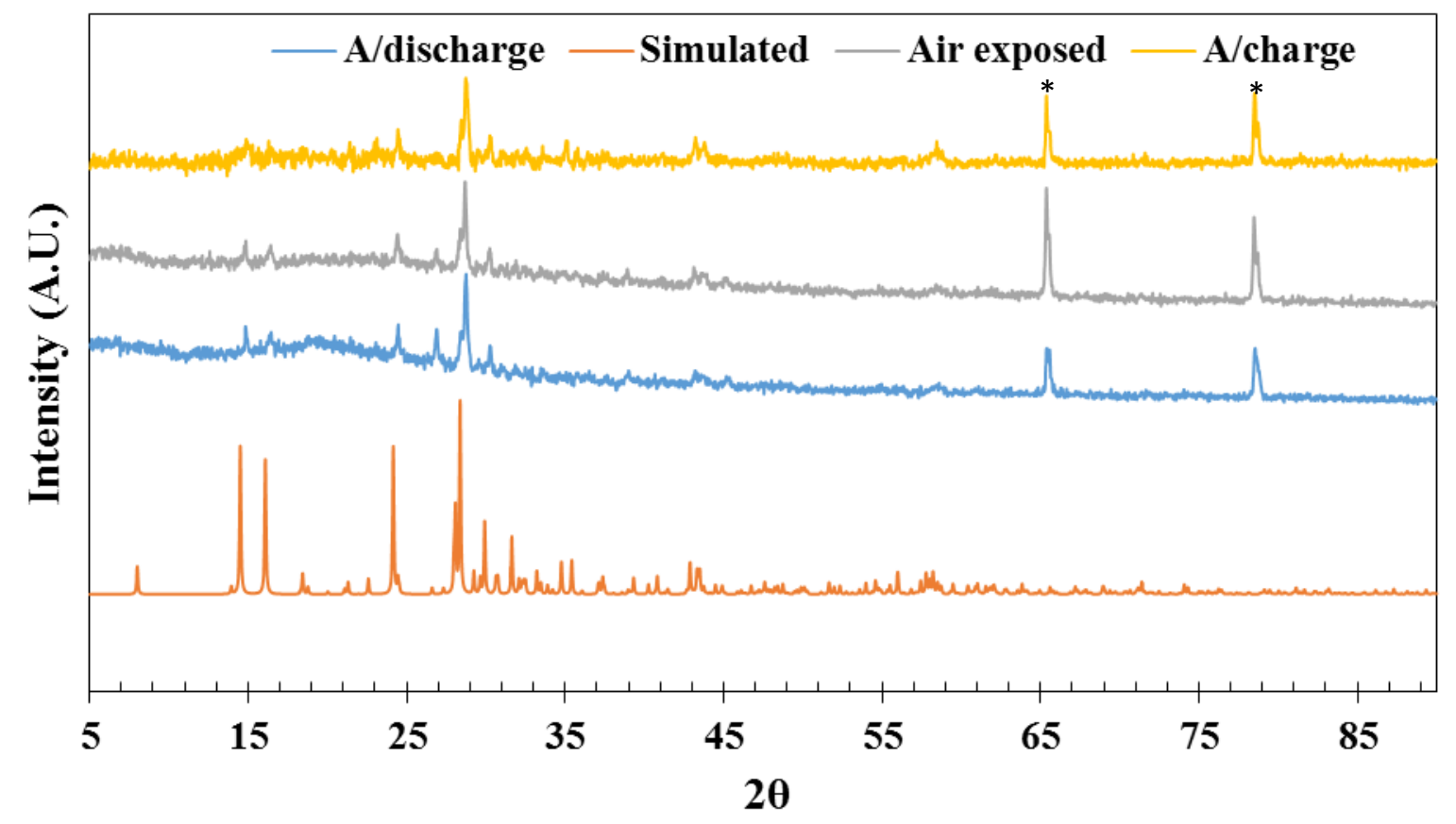

Figure S14. Powder XRD of discharged/charged (in air-tight holder) and air-exposed cathode material for $\mathrm{NaFe}\left(\mathrm{HPO}_{4}\right)_{2}$. (* Aluminum peaks). 


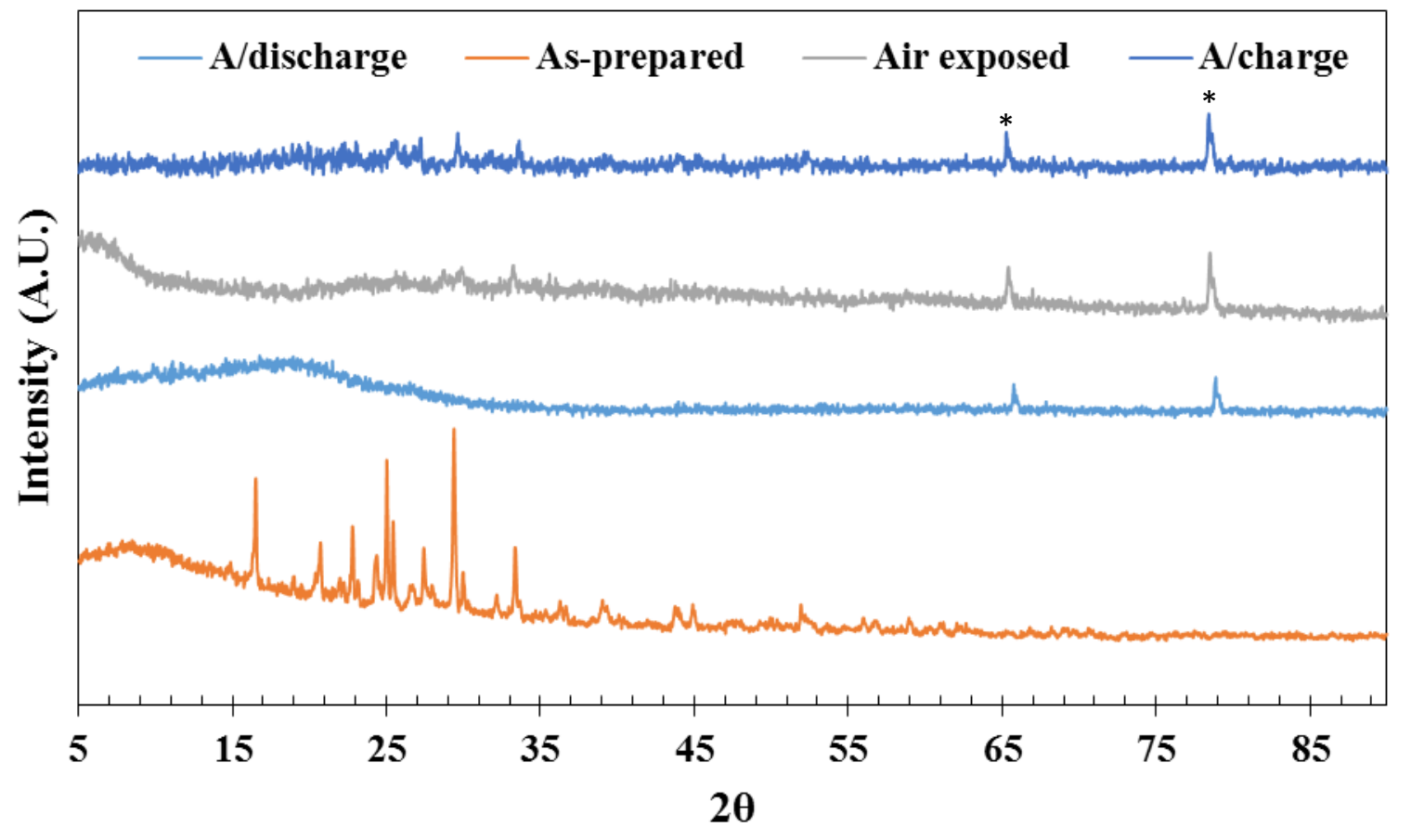

Figure S15. Powder XRD of discharged/charged (in air-tight holder) and air-exposed cathode material for $\mathrm{Li}_{2} \mathrm{Fe}\left(\mathrm{H}_{0.5} \mathrm{PO}_{4}\right)_{2}$-IEX. (* Aluminum peaks). 


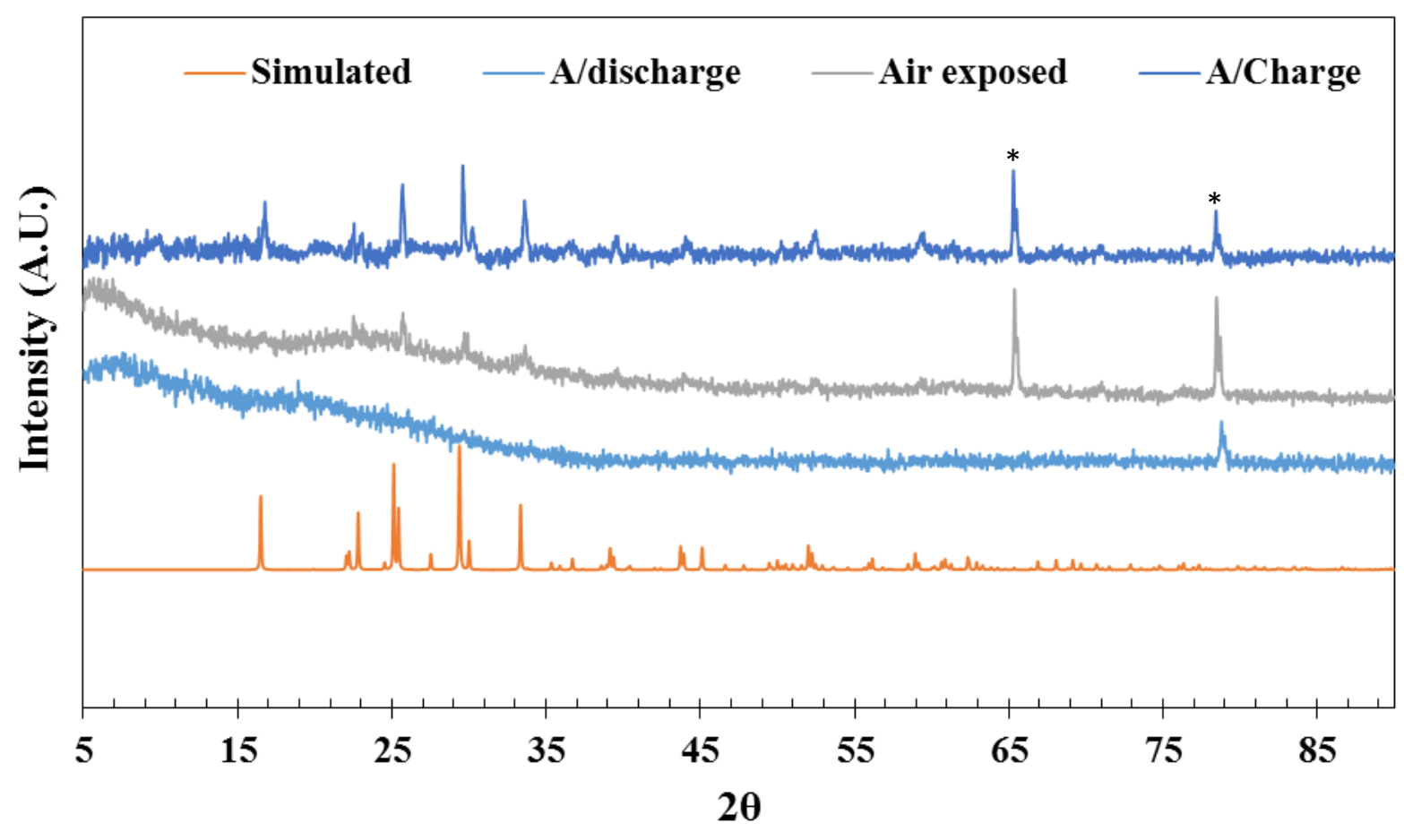

Figure S16. Powder XRD of discharged/charged (in air-tight holder) and air-exposed cathode material for $\mathrm{Li}_{2} \mathrm{Fe}\left(\mathrm{H}_{0.5} \mathrm{PO}_{4}\right)_{2}-\mathrm{HT}$. (* Aluminum peaks). 


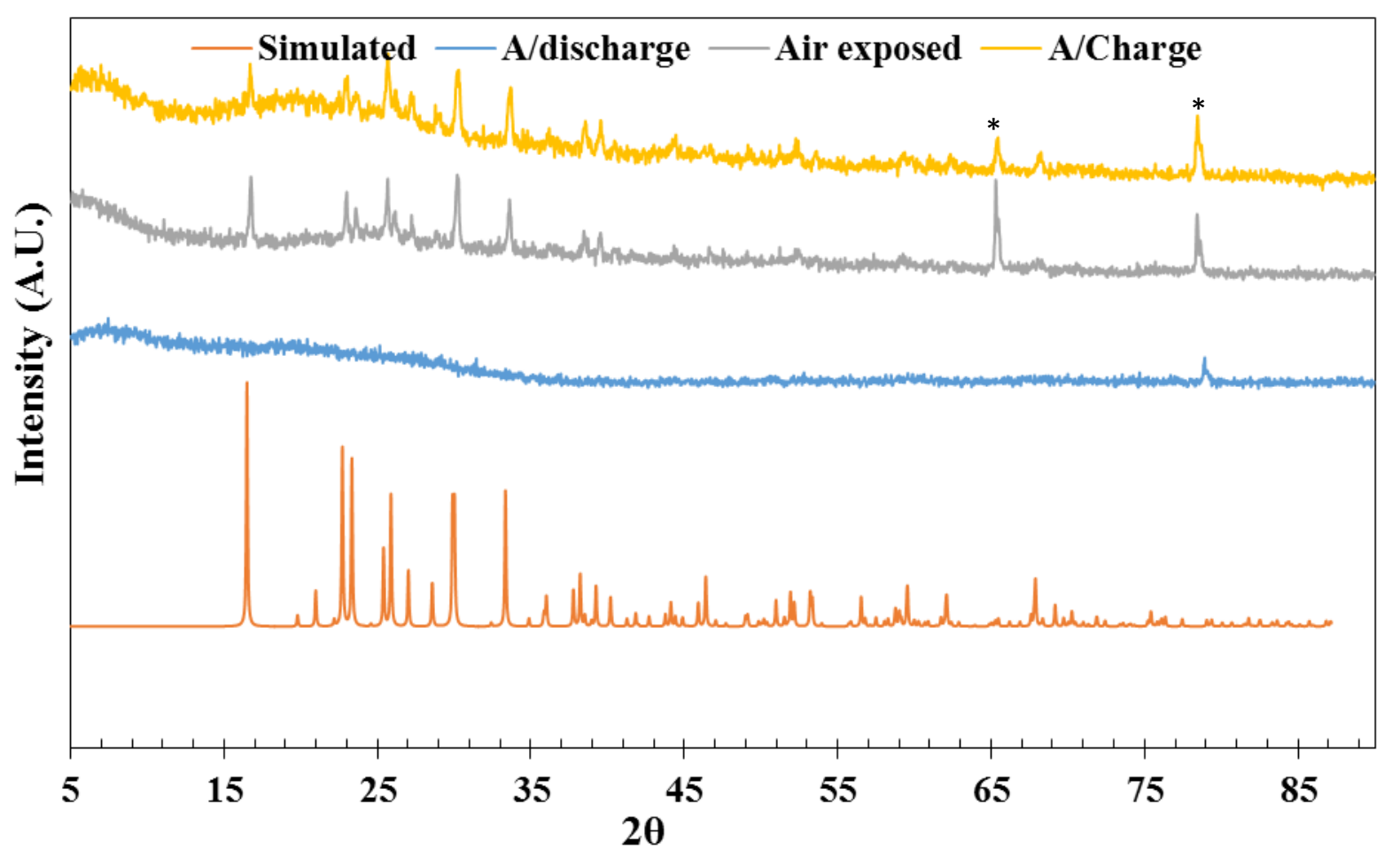

Figure S17. Powder XRD of discharged/charged (in air-tight holder) and air-exposed cathode material for $\mathrm{Li}_{3} \mathrm{Fe}\left(\mathrm{PO}_{4}\right)_{2}$. (* Aluminum peaks). 
Table S1. Atomic coordinates (x $\left.10^{4}\right)$ and equivalent isotropic displacement parameters $\left(\AA^{2} \times 10^{3}\right)$ for $\mathrm{NaFe}\left(\mathrm{HPO}_{4}\right)_{2}$. U(eq) is defined as one third of the trace of the orthogonalized Uij tensor.

\begin{tabular}{|c|c|c|c|c|c|}
\hline \multicolumn{6}{|c|}{$\mathrm{NaFe}\left(\mathrm{HPO}_{4}\right)_{2}$} \\
\hline Atoms & $\mathrm{x}$ & $y$ & $\mathrm{z}$ & $\mathrm{U}(\mathrm{eq})$ & Wyckoff \\
\hline $\mathrm{Fe} 1$ & $9083(1)$ & $5678(1)$ & $8660(1)$ & $6(1)$ & $4 a$ \\
\hline $\mathrm{Fe} 2$ & $7109(1)$ & $8236(1)$ & $6821(1)$ & $6(1)$ & $4 a$ \\
\hline $\mathrm{Fe} 3$ & 5001(1) & $5619(1)$ & $5000(1)$ & $6(1)$ & $4 a$ \\
\hline P1 & $7712(2)$ & $7945(2)$ & $5351(1)$ & $6(1)$ & $4 a$ \\
\hline P2 & $5868(2)$ & $3715(2)$ & $8628(1)$ & $6(1)$ & $4 a$ \\
\hline P3 & $6413(2)$ & $8020(2)$ & 8301(1) & $6(1)$ & $4 a$ \\
\hline P4 & $8172(2)$ & $3636(2)$ & $5022(1)$ & $6(1)$ & $4 a$ \\
\hline P5 & $5144(2)$ & $5417(2)$ & $6535(1)$ & $7(1)$ & $4 a$ \\
\hline P6 & $9927(2)$ & 5913(2) & 7161(1) & $7(1)$ & $4 a$ \\
\hline $\mathrm{O} 1$ & $6747(5)$ & $6979(5)$ & $4959(2)$ & $9(1)$ & $4 a$ \\
\hline $\mathrm{O} 2$ & $8167(6)$ & 9204(5) & 4974(2) & $8(1)$ & $4 a$ \\
\hline $\mathrm{O} 3$ & 6844(5) & $8343(5)$ & $5932(2)$ & $7(1)$ & $4 a$ \\
\hline $\mathrm{O} 4$ & $9297(5)$ & $7178(5)$ & $5550(2)$ & $9(1)$ & $4 a$ \\
\hline O5 & 7616(5) & 4071(5) & $8636(2)$ & $9(1)$ & $4 a$ \\
\hline O6 & $4868(5)$ & 4472(5) & $9086(2)$ & $8(1)$ & $4 a$ \\
\hline $\mathrm{O} 7$ & $5717(5)$ & 2151(5) & $8660(2)$ & $8(1)$ & $4 a$ \\
\hline $\mathrm{O} 8$ & $5304(6)$ & $4218(5)$ & 7977(2) & $10(1)$ & $4 a$ \\
\hline O9 & $7333(6)$ & $7007(5)$ & $8688(2)$ & $9(1)$ & $4 a$ \\
\hline $\mathrm{O} 10$ & $5897(5)$ & $9247(5)$ & $8680(2)$ & $9(1)$ & $4 a$ \\
\hline
\end{tabular}




\begin{tabular}{|c|c|c|c|c|c|}
\hline O11 & $7323(5)$ & $8443(5)$ & $7731(2)$ & $9(1)$ & $4 a$ \\
\hline $\mathrm{O} 12$ & $4828(6)$ & $7266(5)$ & $8075(2)$ & 11(1) & $4 a$ \\
\hline O13 & $6442(5)$ & $4032(5)$ & 4990(2) & $9(1)$ & $4 a$ \\
\hline O14 & $8333(5)$ & $2075(5)$ & $4975(2)$ & $10(1)$ & $4 a$ \\
\hline O15 & $9212(5)$ & $5605(5)$ & $9561(2)$ & $7(1)$ & $4 a$ \\
\hline O16 & $8722(6)$ & $4101(5)$ & $5679(2)$ & 11(1) & $4 a$ \\
\hline O17 & 4911(6) & $5739(5)$ & $5883(2)$ & $12(1)$ & $4 a$ \\
\hline O18 & $8671(5)$ & $9810(5)$ & $6833(2)$ & $9(1)$ & $4 a$ \\
\hline O19 & $5604(5)$ & $6682(5)$ & $6916(2)$ & $9(1)$ & $4 a$ \\
\hline $\mathrm{O} 20$ & $6514(5)$ & $4313(4)$ & $6596(2)$ & $13(1)$ & $4 a$ \\
\hline $\mathrm{O} 21$ & $5235(5)$ & $9540(5)$ & $6845(2)$ & $8(1)$ & $4 a$ \\
\hline $\mathrm{O} 22$ & $8983(5)$ & $6901(4)$ & $6759(2)$ & $8(1)$ & $4 a$ \\
\hline $\mathrm{O} 23$ & $9058(6)$ & $5678(5)$ & $7758(2)$ & $8(1)$ & $4 a$ \\
\hline $\mathrm{O} 24$ & $6620(5)$ & $1566(4)$ & $7293(2)$ & $10(1)$ & $4 a$ \\
\hline $\mathrm{Na} 1$ & $7820(3)$ & $3606(2)$ & $7479(1)$ & $14(1)$ & $4 a$ \\
\hline $\mathrm{Na} 2$ & $8129(4)$ & $8752(3)$ & $3895(1)$ & $20(1)$ & $4 a$ \\
\hline $\mathrm{Na} 3$ & $5964(4)$ & $8911(3)$ & $9816(2)$ & $20(1)$ & $4 a$ \\
\hline
\end{tabular}


Table S2. Atomic coordinates $\left(\mathrm{x} 10^{4}\right)$ and equivalent isotropic displacement parameters $\left(\AA^{2} \times 10^{3}\right)$ for $\mathrm{Li} 2 \mathrm{Fe}\left(\mathrm{H}_{0.5} \mathrm{PO}_{4}\right)_{2}-\mathrm{HT}$. $\mathrm{U}(\mathrm{eq})$ is defined as one third of the trace of the orthogonalized Uij tensor.

\begin{tabular}{llllll}
\hline \multicolumn{5}{c}{$\mathrm{Li} 2 \mathrm{Fe}\left(\mathrm{H}_{0.5} \mathrm{PO}_{4}\right)_{2}-\mathrm{HT}$} \\
\hline Atoms & $\mathrm{x}$ & $\mathrm{y}$ & $\mathrm{z}$ & $\mathrm{U}(\mathrm{eq})$ & Wyckoff \\
\hline $\mathrm{Fe} 1$ & 0 & 0 & 0 & $6(1)$ & $2 a$ \\
$\mathrm{P} 1$ & $6335(1)$ & $6540(1)$ & $7652(1)$ & $6(1)$ & $4 e$ \\
$\mathrm{O} 1$ & $2979(3)$ & $6763(2)$ & $6511(2)$ & $9(1)$ & $4 e$ \\
O2 & $7937(4)$ & $8225(2)$ & $8136(2)$ & $10(1)$ & $4 e$ \\
O3 & $7762(4)$ & $5465(2)$ & $6594(2)$ & $10(1)$ & $4 e$ \\
O4 & $6900(4)$ & $5605(2)$ & $9478(2)$ & $11(1)$ & $4 e$ \\
Li1 & $479(10)$ & $8656(6)$ & $6578(6)$ & $16(1)$ & $4 e$ \\
\hline
\end{tabular}


Table S3. Set of reactions carried out at different $\mathrm{pH}$, temperature and other different conditions.

\begin{tabular}{|c|c|c|c|}
\hline S.No. & Reactants & Conditions & Product (s) \\
\hline 1 & $\begin{array}{l}\mathrm{FeSO}_{4} \cdot 7 \mathrm{H}_{2} \mathrm{O}+\mathrm{H}_{3} \mathrm{PO}_{4}+\mathrm{H}_{3} \mathrm{BO}_{3}+\mathrm{NaF}+ \\
\mathrm{H}_{2} \mathrm{O}\end{array}$ & $\begin{array}{l}185^{\circ} \mathrm{C}, 24 \text { h- } 6 \text { days, } \\
\text { HT }\end{array}$ & $\mathrm{NaFe}\left(\mathrm{HPO}_{4}\right)_{2} *$ \\
\hline 2 & $\mathrm{FeSO}_{4} \cdot 7 \mathrm{H}_{2} \mathrm{O}+\mathrm{H}_{3} \mathrm{PO}_{4}+\mathrm{NaF}+\mathrm{H}_{2} \mathrm{O}$ & $185^{\circ} \mathrm{C}, 24 \mathrm{~h}, \mathrm{HT}$ & $\mathrm{Na}_{3} \mathrm{FeF}_{6}$ \\
\hline 3 & $\begin{array}{l}\mathrm{FeSO}_{4} \cdot 7 \mathrm{H}_{2} \mathrm{O}+\mathrm{H}_{3} \mathrm{PO}_{4}+\mathrm{CH}_{3} \mathrm{COOH}+ \\
\mathrm{NaF}+\mathrm{H}_{2} \mathrm{O}\end{array}$ & $185^{\circ} \mathrm{C}, 24 \mathrm{~h}, \mathrm{HT}$ & $\begin{array}{l}\mathrm{Na}_{3} \mathrm{FeF}_{6} \& \\
\mathrm{Na}_{3} \mathrm{Fe}_{2}\left(\mathrm{PO}_{4}\right)_{2}(\mathrm{OH})_{2} \mathrm{~F}\end{array}$ \\
\hline 5 & $\begin{array}{l}\mathrm{FeSO}_{4} \cdot 7 \mathrm{H}_{2} \mathrm{O}+\mathrm{H}_{3} \mathrm{PO}_{4}+\mathrm{H}_{3} \mathrm{BO}_{3}+\mathrm{LiF}+ \\
\mathrm{H}_{2} \mathrm{O}\end{array}$ & $185^{\circ} \mathrm{C}, 24 \mathrm{~h}, \mathrm{HT}$ & $\mathrm{LiFe}\left(\mathrm{PO}_{4}\right)(\mathrm{OH})_{\mathrm{X}} \mathrm{F}_{1-\mathrm{x}}$ \\
\hline 6 & $\begin{array}{l}\mathrm{FeSO}_{4} \cdot 7 \mathrm{H}_{2} \mathrm{O}+\mathrm{H}_{3} \mathrm{PO}_{4}+\mathrm{H}_{3} \mathrm{BO}_{3}+\mathrm{NaOH} \\
+\mathrm{H}_{2} \mathrm{O}\end{array}$ & $185^{\circ} \mathrm{C}, 24 \mathrm{~h}, \mathrm{HT}$ & $\mathrm{NaFeBP}_{2} \mathrm{O}_{7}(\mathrm{OH})_{3}$ \\
\hline 7 & $\begin{array}{l}\mathrm{FeSO}_{4} \cdot 7 \mathrm{H}_{2} \mathrm{O}+\mathrm{H}_{3} \mathrm{PO}_{4}+\mathrm{H}_{3} \mathrm{BO}_{3}+\mathrm{NaF}+ \\
\mathrm{H}_{2} \mathrm{O}\end{array}$ & $145^{\circ} \mathrm{C}, 24 \mathrm{~h}, \mathrm{HT}$ & No solid product \\
\hline 9 & $\mathrm{NaFe}\left(\mathrm{HPO}_{4}\right)_{2}+\mathrm{LiNO}_{3}+\mathrm{H}_{2} \mathrm{O}$ & $185^{\circ} \mathrm{C}, 24 \mathrm{~h}, \mathrm{HT}$ & $\mathrm{LiFe}\left(\mathrm{PO}_{4}\right)(\mathrm{OH})$ \\
\hline 10 & $\mathrm{NaFe}\left(\mathrm{HPO}_{4}\right)_{2}+\mathrm{LiNO}_{3}+\mathrm{H}_{2} \mathrm{O}$ & $75^{\circ} \mathrm{C}, 8 \mathrm{~h}, \mathrm{IEx}$, stir & $\mathrm{NaFe}\left(\mathrm{HPO}_{4}\right)_{2}$ \\
\hline 11 & $\mathrm{NaFe}\left(\mathrm{HPO}_{4}\right)_{2}+\mathrm{LiNO}_{3}+\mathrm{H}_{2} \mathrm{O}$ & $75^{\circ} \mathrm{C}, 16 \mathrm{~h}, \mathrm{IEx}$, Stir & $\mathrm{NaFe}\left(\mathrm{HPO}_{4}\right)_{2}$ \\
\hline 12 & $\mathrm{NaFe}\left(\mathrm{HPO}_{4}\right)_{2}+\mathrm{LiNO}_{3}+\mathrm{H}_{2} \mathrm{O}$ & $75^{\circ} \mathrm{C}, 24 \mathrm{~h}, \mathrm{IEx}$, Stir & $\begin{array}{l}\mathrm{NaFe}\left(\mathrm{HPO}_{4}\right)_{2}+ \\
\mathrm{Li}{ }_{2} \mathrm{Fe}\left(\mathrm{H}_{0.5} \mathrm{PO}_{4}\right)_{2}\end{array}$ \\
\hline 13 & $\mathrm{NaFe}\left(\mathrm{HPO}_{4}\right)_{2}+\mathrm{LiNO}_{3}+\mathrm{H}_{2} \mathrm{O}$ & $75^{\circ} \mathrm{C}, 48$ h, IEx, Stir & $\begin{array}{l}\mathrm{Li} 2 \mathrm{Fe}\left(\mathrm{H}_{0.5} \mathrm{PO}_{4}\right)_{2} \\
\text { (major) \& } \\
\mathrm{Fe}\left(\mathrm{PO}_{4}\right) \cdot 2 \mathrm{H}_{2} \mathrm{O} \text { (minor) }\end{array}$ \\
\hline 14 & $\mathrm{Fe}\left(\mathrm{NO}_{3}\right)_{3} \cdot 9 \mathrm{H}_{2} \mathrm{O}+\mathrm{LiOH} \cdot \mathrm{H}_{2} \mathrm{O}+\mathrm{H}_{3} \mathrm{PO}_{3}$ & $175^{\circ} \mathrm{C}, 6$ days & $\mathrm{Li}_{2} \mathrm{Fe}\left(\mathrm{H}_{0.5} \mathrm{PO}_{4}\right)_{2} *$ \\
\hline 15 & $\mathrm{Li}_{2} \mathrm{Fe}\left(\mathrm{H}_{0.5} \mathrm{PO}_{4}\right)_{2}+\mathrm{LiNO}_{3}$ & $\begin{array}{l}215^{\circ} \mathrm{C}, 3 \text { days Solid } \\
\text { state IEX }\end{array}$ & $\mathrm{Li}_{3} \mathrm{Fe}\left(\mathrm{PO}_{4}\right)_{2} *$ \\
\hline
\end{tabular}

*This work 